International Journal of Mechanical Engineering and Technology (IJMET)

Volume 11, Issue 12, December 2020, pp. 12-18, Article ID: IJMET_11_12_002

Available online at https://iaeme.com/Home/issue/IJMET?Volume $=11 \&$ Issue $=12$

ISSN Print: 0976-6340 and ISSN Online: 0976-6359

DOI: https://doi.org/10.34218/IJMET.11.12.2020.002

(C) IAEME Publication

Scope Database Indexed

\title{
EFFECT OF TYRE UNBALANCE ON PERFORMANCE OF VEHICLE
}

\author{
Sanjay Taneja \\ Department of Mechanical Engineering, Manav Rachna University, Aravalli Hills, \\ Manav Rachna Campus Rd, Faridabad, Haryana, India.
}

\begin{abstract}
Now a days automobiles play a significant role in our day to day life, but automobile vehicles (cars) also need proper care and service to get optimum performance from them. Balancing technology is one of the important aspect of automobile vehicle and unbalance in tyres of vehicle plays an important part in vehicle performance.

Of course, it's natural for any rotating component to vibrate. Even in the best of operating condition vehicle will have some minimum vibration because of small, minor defects. However, when machinery vibration increases or becomes excessive, some mechanical trouble is usually the reason, which can be - unbalance, misalignment, worn out gears or bearings, looseness, etc.

This paper focusses importance of balancing technology specially effect of tyre unbalance and its adverse effect on vehicle (car) performance and remedial steps to improve these effects. Idea of writing this type of paper is to highlight the significance of balancing technology in a high speed vehicles (specially cars) and also to aware the consumers (car owners) and manufacturer both about this paramount technology.

Sometimes these studies are quite helpful in order to facilitate better design of wheel assembly (which includes tyres and wheel both) so that there should be minimum unbalance which affects the performance of vehicle.
\end{abstract}

Key words: PIA- Principle inertia axis, Quality grade, Tread, Un-sprung weight, Understeer, Wheel assembly (wheel and tyre fitted together)

Cite this Article: Sanjay Taneja, Effect of Tyre Unbalance on Performance of Vehicle, International Journal of Mechanical Engineering and Technology. 11(12), 2020, pp. 12-18.

https://iaeme.com/Home/issue/IJMET?Volume=11\&Issue $=12$

\section{INTRODUCTION}

Unbalance in a machine or in any moving part designates the lack of equilibrium in the masses of a solid rotating about its axis. Identifying and correcting the mass distribution and thus minimizing the force and resultant vibration is the technique known as balancing. 
If unbalance exists in vehicle tyre, it means wheel assembly tends to vibrate, causing damage to tyre (treads or uneven wear) and vehicle parts like bearings, suspension, steering parts etc. Research showed that the wheel, Tyre and its axle assembly had to be in a state of balance. The distribution of mass had to be even about the rotating centreline so that the resultant vibration is at a minimum. This had to be achieved during the designing \& manufacturing process so that maximum service life could be achieved. As vehicles go faster, the effect of the unbalance is much more severe. A level of unbalance that is acceptable at a low speed is completely unacceptable at a higher speed. This is because the unbalance condition produces centrifugal force, which increases as the speed increases. It is the force due to unbalance that actually causes noise and vibration of the surrounding parts. Prolonged exposure to the vibration results in damage due to increased Noise \& discomfort. Vibration can also be transmitted to adjacent part, affecting their accuracy or performance. The ultimate goal is to produce a tyre with as small unbalance as an economically feasible. So by controlling the process variation the level of unbalance within the acceptable limit as described in ISO standard 1940 (by selecting appropriate Quality grades) [2, 3, 6].

Practically, vibrations cannot reach zero values but usually it is acceptable to decrease them to a value lower than that one prescribed for a certain quality class of the machinery as mentioned in ISO standard 1940 [2, 3]. It should also be ensured that wheel assembly (wheel and tyre) unbalance should be well within the acceptable limits.

This paper focusses effects of unbalanced Tyre on performance of vehicle and also highlights remedial steps to be taken to avoid these problems.

\section{THEORY OF UNBALANCE}

The uneven distribution of mass about a rotor's rotating centreline is Unbalance. The rotating centreline being defined as the axis about which the rotor would rotate if not constrained by its bearings also called the Principal Inertia Axis (PIA). The geometric centreline being the physical centreline of the rotor. When the two centrelines are coincident, then the rotor will be in a state of balance. When they are apart, the rotor will be unbalanced.

Static Unbalance - where the Principal Inertia Axis (PIA) is displaced parallel to the geometric centre line. Force unbalance is another name of static unbalance and it requires only one correction with a known weight at particular angle.

Couple Unbalance - where the Principal Inertia Axis (PIA) intersects the geometric centre line at the centre of gravity (CG). It requires two equal weights to the work piece at angles $180^{\circ}$ apart in two correction planes.

Dynamic Unbalance - where the Principal Inertia Axis (PIA) and the geometric centre line do not coincide or touch. A wheel is in dynamic balance, then it is also in static balance. However, a wheel can be in static balance without necessarily being in dynamic balance.

Sprung weight is the portion of the vehicle's total mass that is supported above the suspension. The mass of the components suspended below the suspension components including the wheels, wheel bearings, brake rotors, callipers, which are part of the vehicle's unsprung weight. The un-sprung weight of a wheel controls a trade-off between a wheel's bumpfollowing ability and its vibration isolation. Bumps and surface imperfections in the road cause tyre compression - which induces a force on the un-sprung weight [13].

A pure unbalance creates a centrifugal force that is radial, and is well defined by the formula:

$\mathrm{Fc}=\mathrm{mr} \omega^{2}$

$\mathrm{Fc}$ is the centrifugal force (Unbalance)

$\mathrm{m}$ is the imaginary mass 
$r$ is the radius of the imaginary mass

$\omega$ is the rotating speed in radians per second.

The concept of an imaginary mass is taken for unbalance because the exact cause is unknown for each specific rotor. It could be caused by non-uniform material density (porosity), or eccentricity from poor fits. When correction weight is applied, then the mass and radius of the correction weight becomes $180^{\circ}$ opposite from this imaginary mass.

The unbalanced centrifugal force has two effects:

- It will distort the rotor.

- It will couple into the stationary structure as an oscillation at the frequency of rotation.

The centrifugal forces are created on the rotating part where the defect is, but the damaging forces are felt at the bearings and the stationary structure.

\section{EFFECT OF TYRE UNBALANCE ON VEHICLE}

\subsection{Vibrations in steering wheel}

One of the earliest warning signs of unbalanced tyres is feeling vibrations in the steering wheel. When the unbalance worsens as the tyres wear out, the vibration will increase and affect the entire cabin of the vehicle, including the floorboards and front and rear seats. Other problems can also cause vibrations, such as a damaged tyre or problems in the wheel assembly, which needs to be rectified at the earliest to avoid any serious problem.

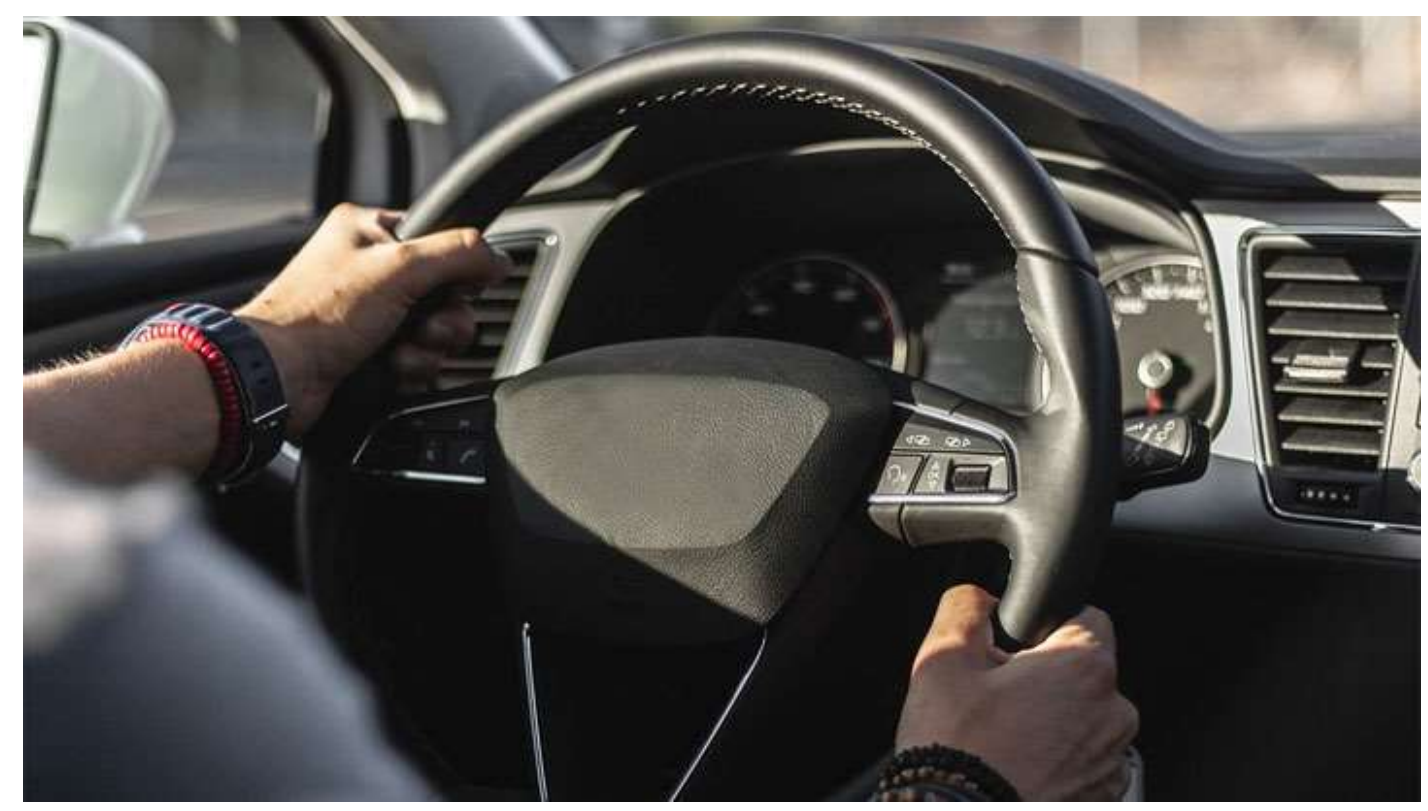

Figure 1. shows Steering wheel vibration

Unbalanced tyres will also create an uncomfortable driving experience by making steering more difficult and delaying the response time of the steering wheel. More importantly, Driver will not be able to steer vehicle smoothly in a given direction or more effort is required to steer the vehicle also known as understeer.

\subsection{Uneven Tyre wear}

It's important to regularly inspect the tyre treads, as one can feel a lot about how well vehicle tyres are balanced. Uneven tyre wear can be caused by unbalanced tyres or by other issues with the steering or wheels. 
The vehicle is at increased risk of having a flat tyre means vehicle can skid if tyres are allowed to wear abnormally. The tyre can thin to the point of spontaneous rupture, which can be a dangerous situation if it happens while driving.

Rotating the vehicle tyres can be a temporary solution until one have the money to replace the ones that are too worn out. New tires that are installed should immediately be balanced to avoid any crunch situation.

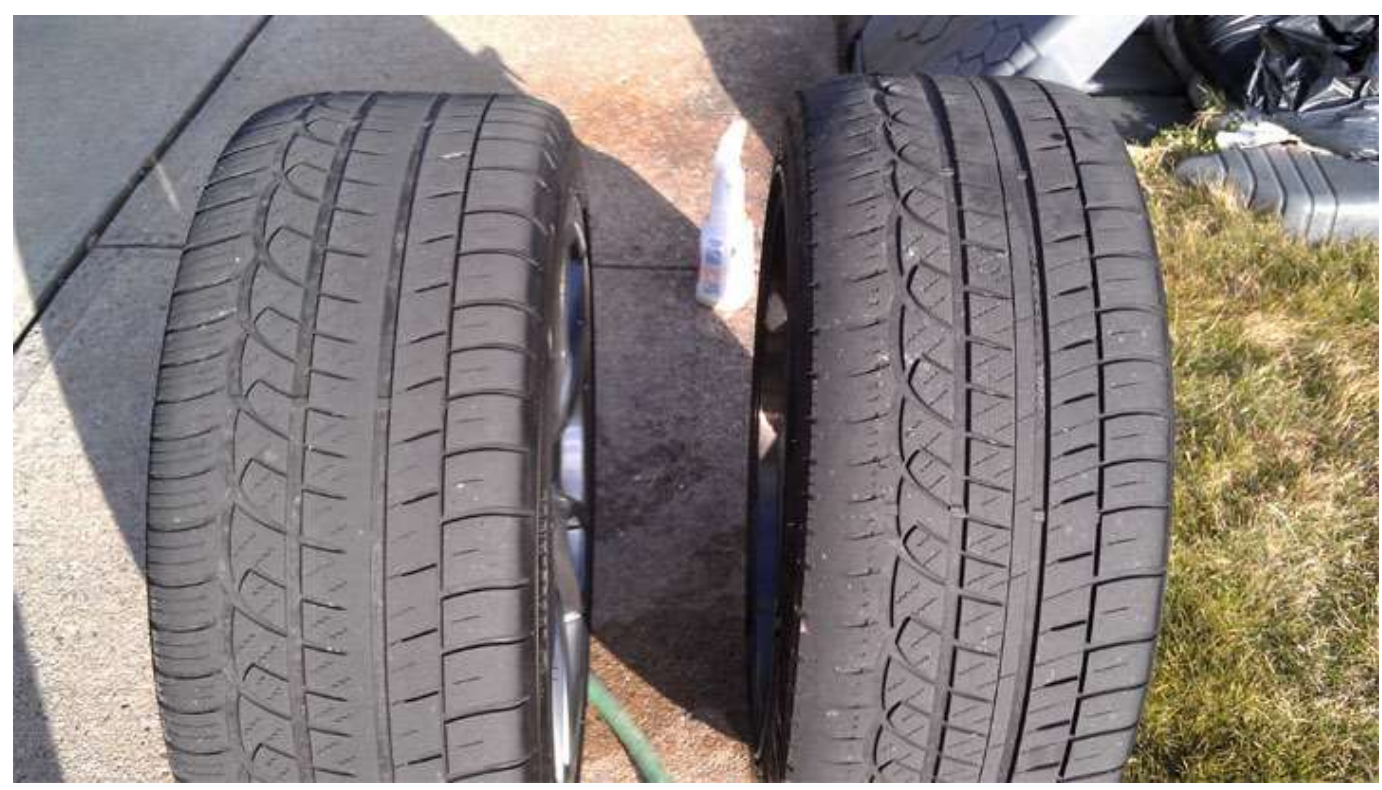

Figure 2. shows pattern of Tyre wear

\subsection{Reduced Fuel Economy}

Unbalanced tyres always put more stress on the engine of your vehicle, since the resistance created by the unbalance forces the engine to work harder just to keep the vehicle in motion. As a result, the engine will require more fuel to sustain its operation under these conditions.

Under-inflated tyres can also cause the vehicle to consume more fuel than usual, hence it is essential to check air pressure by using a tyre pressure gauge at regular intervals.

It's very smart to keep track of vehicle's fuel economy. While some newer cars have a fuel economy indicator, these can be inaccurate or not properly calibrated so it also become important to keep a watch on fuel economy of a vehicle.

\subsection{Uneven Shocks and Bearing life}

The springs, bearings, and other components of the wheel assembly are under a lot of stress when the tyres are not balanced so they will experience more wear-and-tear than normal, it means uneven shocking in vehicle and ultimately it is going to affect bearing life.

\section{REMEDIAL STEPS FOR IMPROVING VEHICLE PERFORMANCE}

\subsection{Balancing of Tyre}

Tyres are balanced when the weight is evenly distributed all around the tyre. It's important to have well-balanced tyres when driving for the best performance, tyre life, and safety. New tyres can have imperfections from the manufacturer, and driving also causes wear on them. Balancing needs to happen each time especially when new tyres are installed and regularly thereafter during tyre rotations for proper maintenance $[8,11,12]$. 


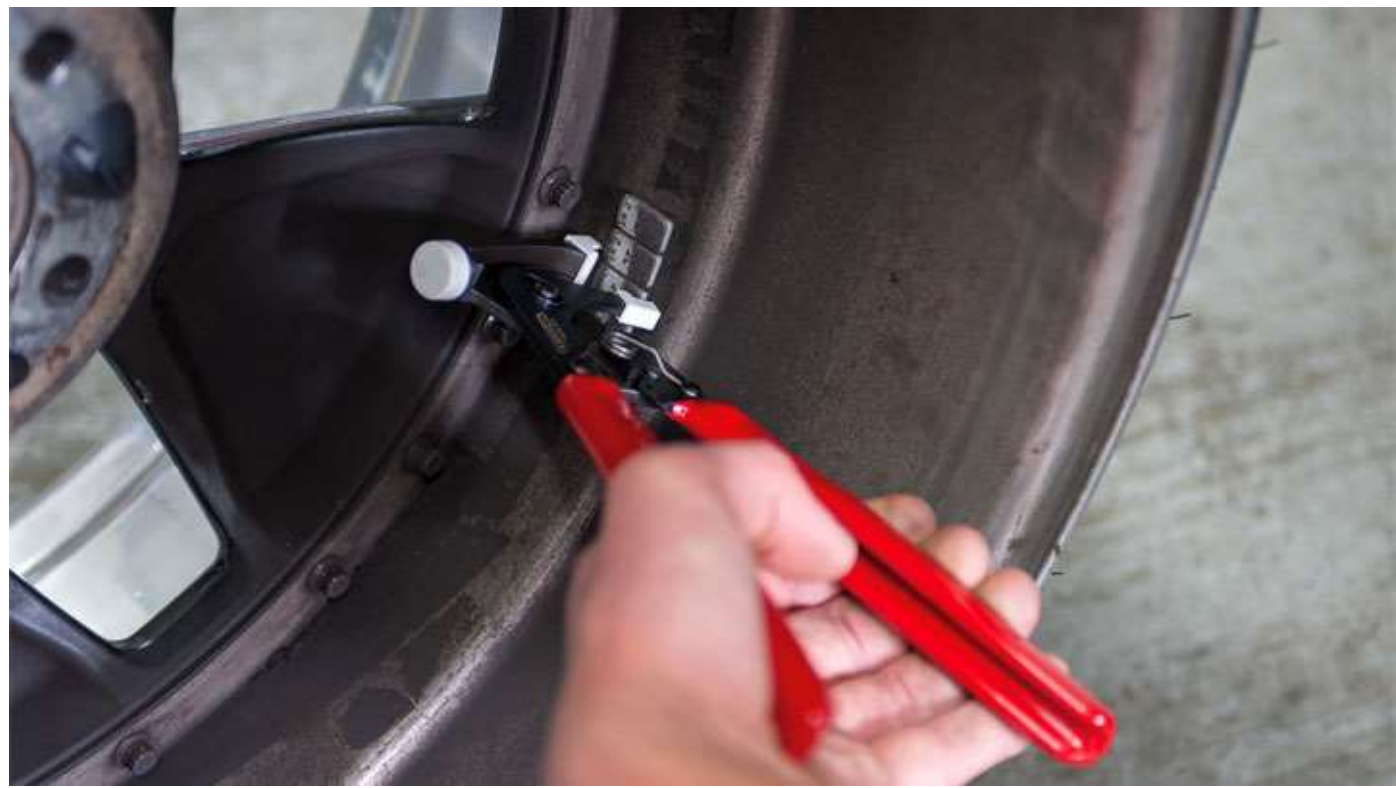

Figure 3. Shows balancing of Tyre

\subsection{Tyre inflation}

The best performance of tyres can only be achieved when the tyre is inflated to the designated pressure based on the load per tyre. "Under inflation" or "Over inflation" on the tyre tend to impact tyre life, vehicle handling and safety. This can cause heat build-up/tyre temperature and thus loss of tyre life, premature tyre removals, increased rolling resistance and fuel consumption. "Under Inflation" is more common than over inflation. Tyre users are not always conscious about maintaining or matching tyre pressure to the loads carried. Check the pressures of your tyres, including spare, once in a fortnight and before any long journey or if traveling with a heavy load. Ensure a dirt-free valve cap is always fitted.

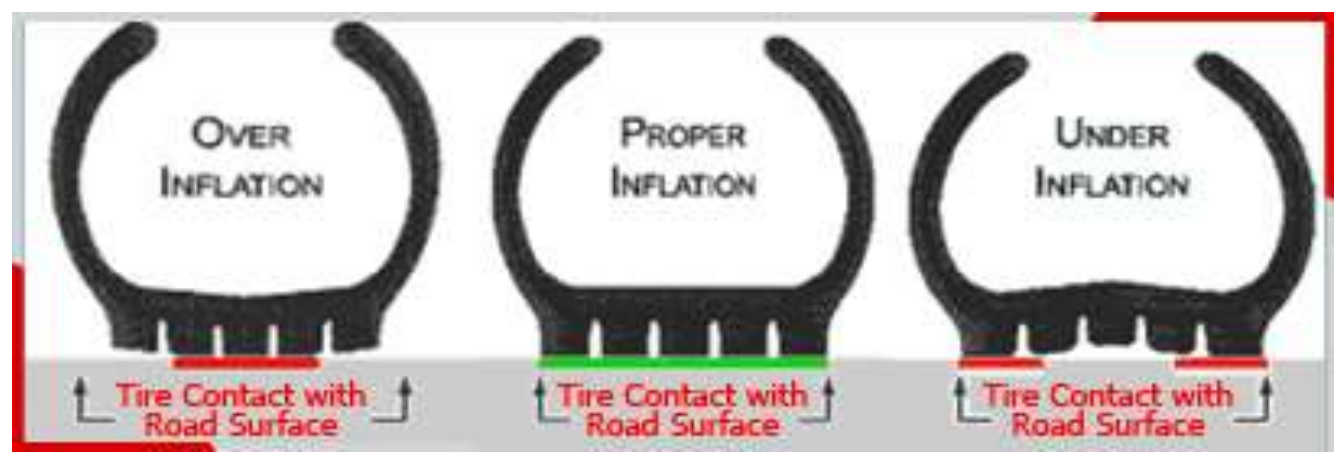

Figure 4. shows contact with road surface

Figure 4 clearly shows that for making a proper contact with road surface tyre always should be properly inflated.

\subsection{Speed}

Excessive high speeds results in increased tyre running temperature. This results in increased tyre wear at a rapid rate and separation of components (treads of tyre).

\subsection{Driving habits}

Careful driving habits will ensure optimum tyre life. Some of the habits which cause serious damages to tyre are rash driving, over speeding, speeding over pot holes, stone, rough roads, quick starts and sudden stops, riding over road divider and other obstacles, sharp turns at high 
speeds, hitting the road, curbs, objects. Running on improperly inflated tyres is also one of the important reason.

\subsection{Road Conditions}

Vehicle/tyre operating conditions significantly influence tyre life both in terms of new tyre life and structural durability, hence it is essential to avoid fast driving on rough roads to prevent early tyre damage.

\subsection{Weather Effects}

Climatic and weather conditions in our country varies widely from region to region. Dry and extremely hot during summer, extreme cold during winter and rains during monsoon. This variation in climatic conditions influence tyre life in terms of mileage and structural durability. It is seen in our country that summer weather is having most adverse impact on life of the tyre which ultimately affect the performance of the whole vehicle.

\section{CONCLUSION}

Actually all rotating components experience significant quality improvements if balanced. In today's scenarios of global market, customers are looking for the best and efficient products available for their money. They demand maximum performance, minimum size, and lower cost. In addition everything must be smaller, more efficient, and more powerful, weigh less, run quieter, smoother and last longer.

As consumer demands continue to increase, balanced components will remain an essential ingredient. Balancing will always be one of the most cost effective means of providing quality products to consumers. Hence all rotating parts needs to be in a state of balance to ensure smooth running while in operation and it also become essential that before designing and manufacturing of any rotating component, engineer must consider balancing aspect for the same.

To achieve best vehicle performance, companies and vehicle owners must ensure perfectly balanced tyre and wheel assembly as per ISO standard with appropriate quality grade $[2,3$, and $6]$.

\section{REFERENCES}

[1] Balance Quality Requirements of Rotating Rigid Bodies. American National Standards Institute. ANSI S2. 19-1975.

[2] Balance Quality Requirements of Rigid Rotors. British Standards Institution. BS 68611:1987. ISO 1940-1:1986

[3] Balance Quality Requirements of Rigid Rotors." International Organization for Standardization. ISO 1940 STANDARD for Balancing Masses1. ISO 1940/1

[4] Shiyu Zhou and Jianjun Shi, -Active Balancing and Vibration Control of Rotating Machinery: A Survey\|, The Shock and Vibration Digest, July 2001, Vol. 33, No. 4, 361371.

[5] Entek IRD International, Introduction to vibration technology. Edition I, Jul 1984, Tata McGraw hills.

[6] Balance Quality Requirements of Rigid Rotors. German Standards Institution. The Practical Application of ISO 1940/1, VDI 2060. 
[7] DYNAMIC BALANCING HANDBOOK, "October 1990, IRD Mechanalysis Inc. ISO 1925, "Balancing Vocabulary." International Organization for Standardization.

[8] "Indian Tyre Technical Advisory Committee" (ITTAC) Standard Manual 2014, Pages R08-R-20.

[9] Jim Lyons "Dynamic Balancing Causes, Corrections and Consequences" Main Tech South '98, 1998, Pages 3-5.

[10] Luigi Buzzi,"Technical Elements of Rotor Balancing", CEMB Technical Booklet N. 3 II Edition 1995, Pages 7-10.

[11] Tananko D, Krivtsov V, Rohwader D, "Do we really need a spec on tyre static balance?" SAE Technical Paper 2003-01-0151. Pages 2-4.

[12] Srinivasan P, "The effect of wheel unbalance on vehicle Dynamics", California Institute of Technology, California, 1956. Pages 17-21.

[13] Bixel, Heydineger, Guenther and Novak, "Sprung/Unsprung Mass Properties Determination without Vehicle Disassembly", SAE Technical Paper 960183, 1996. Page 91. 